\title{
A COHOMOLOGICAL INTERPRETATION OF BOGOMOLOV'S INSTABILITY
}

\author{
GABRIELE DI CERBO
}

(Communicated by Lev Borisov)

\begin{abstract}
We give a new proof of Bogomolov's instability theorem. Furthermore, we prove that it is equivalent to a statement which characterizes when the first cohomology group of a suitable divisor does not vanish.
\end{abstract}

\section{INTRODUCTION}

In the theory of stable vector bundles on surfaces, the following theorem, known as Bogomolov's instability theorem, plays a central role:

Theorem 1.1 (Bogomolov). Let $X$ be a smooth projective surface and $V$ be a rank 2 vector bundle on $X$. If $c_{1}(V)^{2}>4 c_{2}(V)$, then $V$ is unstable.

For the original proof we refer to [1] see also 9 . This theorem was later proved by quite different techniques in [5] and [8]. Furthermore, Reider used Theorem 1.1 to study adjoint linear series on surfaces and to derive his famous theorem, [10].

The first cohomological proof of Reider's theorem was given by Sakai in 11 . His proof uses ideas of Serrano [12] and generalizes Reider's theorem to normal surfaces. The key point in Sakai's proof is the following theorem.

Theorem 1.2 (Sakai). Let $D$ be a big divisor with $D^{2}>0$ on a smooth projective surface $X$. If $H^{1}\left(X, \mathcal{O}_{X}\left(K_{X}+D\right)\right) \neq 0$, then there exists an effective divisor $E$ such that

(1) $D-2 E$ is big;

(2) $(D-E) \cdot E \leq 0$.

As shown in 11, Theorem 1.2 can be easily derived from Theorem 1.1. Moreover, Sakai gave an alternative proof based on Miyaoka's vanishing theorem for the Zariski decomposition of a divisor. Later Ein and Lazarsfeld showed how to apply the Kawamata-Viehweg vanishing theorem to prove a part of Reider's theorem in [2. Based on these new techniques Fernández del Busto gave an elegant proof of Bogomolov's inequality which uses only the Kawamata-Viehweg theorem; see 3 . For a survey on these results we refer to [6].

On the other hand, Mumford showed that we can use Bogomolov's theorem for rank 2 vector bundles to give a short proof of a generalized Kodaira vanishing for surfaces; see 44. This vanishing theorem is a little less general than the theorem of Kawamata-Viehweg. These results suggest that there should be a connection between Bogomolov's instability and some vanishing theorem.

Received by the editors September 28, 2011 and, in revised form, December 4, 2011.

2010 Mathematics Subject Classification. Primary 14F17, 14J60. 
In this note we prove

Theorem 1.3. Bogomolov's instability theorem is equivalent to Theorem 1.2 .

Furthermore, using Sakai's proof of Theorem 1.2, one gets a new proof of Bogomolov's instability theorem which is entirely cohomological.

We now outline the proof of Theorem 1.3. After twisting the vector bundle $V$ with a line bundle, we can assume that $V$ has a global section. Using this section we have that the extension class of the vector bundle is nontrivial since $V$ is locally free. The first step of our proof follows Fernández del Busto's argument [3]. At this point we follow a different strategy. The numerical condition of Bogomolov's inequality allows us to apply Theorem [1.2, and we show directly that the divisor $E$ gives the destabilizing subsheaf.

\section{Preliminaries}

For the convenience of the reader we sketch the proof of Theorem 1.2

Proof. Let $D=P+N$ be the Zariski decomposition of $D$ and write $N=\sum \alpha_{j} E_{j}$ with each $\alpha_{j}$ positive and rational. By Sakai's lemma, Example 9.4.12 in [7, we know that $H^{1}\left(X, \mathcal{O}_{X}\left(K_{X}+D-\lfloor N\rfloor\right)\right)=0$ so $\lfloor N\rfloor>0$. Consider the following sequence of divisors:

$$
D_{0}=D-\lfloor N\rfloor, \ldots, D_{k}=D_{k-1}+E_{j_{k}}, \ldots, D_{n}=D .
$$

If $D_{k} \cdot E_{j_{k}}>0$ for any $k$, we get the vanishing of $H^{1}\left(X, \mathcal{O}_{X}\left(K_{X}+D\right)\right)$. Thus we can collect all the $E_{j_{k}}$ 's with positive intersection to construct a sequence $D_{0}, \ldots, D_{k}$ such that $\left(D-D_{k}\right) \cdot E_{j} \leq 0$ for all irreducible components $E_{j}$ of $D-D_{k}$. Now a computation shows that $E:=D-D_{k}$ is the required divisor.

Corollary 2.1. Let $D$ and $E$ be as above. Then

$$
H^{1}\left(X, \mathcal{O}_{X}\left(K_{X}+D-E\right)\right)=0 .
$$

Proof. By the above construction,

$$
H^{1}\left(X, K_{X}+D_{0}\right)=H^{1}\left(X, K_{X}+D_{k}\right) .
$$

Since $D_{0}=D-\lfloor N\rfloor$ and $D_{k}=D-E$, the result follows from Sakai's lemma.

In conclusion we recall two results which will be used in the proof of the main theorem.

Lemma 2.2. Let $f: Y \rightarrow X$ be a birational morphism between smooth projective surfaces and $\widetilde{L}$ a divisor on $Y$. Set $L:=f_{*} \widetilde{L} ;$ if $\widetilde{L}^{2}>0$ and $L$ is big, then $\widetilde{L}$ is big.

Proof. Lemma 3 in [1].

Proposition 2.3. Let $f: \widetilde{X} \rightarrow X$ be a birational morphism between smooth projective surfaces. Let $\widetilde{D}$ be a divisor on $\widetilde{X}$ such that $\widetilde{D}^{2}>0$. Suppose there is a divisor $\widetilde{E}$ which satisfies the conclusions of Theorem 1.2 and let $D:=f_{*} \widetilde{D}, E:=f_{*} \widetilde{E}$ and $\alpha:=D^{2}-\widetilde{D}^{2}$. If $D$ is nef and $E$ is effective, we have

$$
0 \leq D \cdot E<\alpha / 2 .
$$

Proof. See Proposition 2 in [1]. 


\section{MAIN THEOREM}

We can now prove the main result of the paper.

Proof of Theorem 1.3. As mentioned before, Theorem 1.2 can be easily proved using Bogomolov's instability; see [11, p. 307.

We now want to show that Theorem 1.2 implies Bogomolov's theorem. Since the inequality in Theorem 1.1 is invariant under twisting with a line bundle, we can assume that $V$ is globally generated, $\operatorname{det}(V)$ is ample and $c_{2}(V)>0$. Taking a general section $s$ of $V$, we get the following exact sequence:

$$
0 \rightarrow \mathcal{O}_{X} \rightarrow V \rightarrow L \otimes I_{Z} \rightarrow 0
$$

where $L:=\operatorname{det}(V)$ and $Z$ is the zero locus of $s$. Then we have $c_{2}(V)=|Z|$, the length of $Z$.

Since $V$ is locally free, the above extension is nontrivial and then

$$
H^{1}\left(X, \mathcal{O}_{X}\left(K_{X}+L\right) \otimes I_{Z}\right) \neq 0 .
$$

Let $\pi: Y \rightarrow X$ be the blow up of $X$ at all points in $Z$. Let $E_{j}$ be the exceptional curve over $x_{j} \in Z$. Then

$$
H^{1}\left(Y, \mathcal{O}_{Y}\left(K_{Y}+\pi^{*} L-2 \sum_{j} E_{j}\right)\right)=H^{1}\left(X, \mathcal{O}_{X}\left(K_{X}+L\right) \otimes I_{Z}\right) \neq 0 .
$$

Define $\widetilde{L}:=\pi^{*} L-2 \sum_{j} E_{j}$. Thus, we have

$$
\widetilde{L}^{2}=\left(\pi^{*} L\right)^{2}+4 \sum_{j} E_{j}^{2}=c_{1}^{2}(V)-4 c_{2}(V)>0,
$$

so $\widetilde{L}$ is big by Lemma 2.2

By applying Theorem 1.2 we get an effective divisor $\widetilde{E}_{s}$ such that

(1) $\widetilde{L}-2 \widetilde{E}_{s}$ is big;

(2) $\left(\widetilde{L}-\widetilde{E}_{s}\right) \cdot \widetilde{E}_{s} \leq 0$.

Note that $\widetilde{E}_{s}$ depends on the section $s$ that we chose at the beginning. Let $E_{s}:=$ $\pi_{*} \widetilde{E}_{s}$. We want to show that, for any $s, E_{s}$ passes through at least one point of $Z$. Let $\widetilde{E}_{s}:=\pi^{*} E_{s}+\sum a_{i} E_{i}$, where $E_{i}$ are the exceptional divisors. It suffices to show that there exists an index $i$ such that $a_{i}<0$. Write $\widetilde{L}-\widetilde{E}_{s}=\pi^{*} W_{s}-\sum\left(a_{i}+2\right) E_{i}$, where $W_{s}:=L-E_{s}$. Thus by (2) we have

$$
E_{s} \cdot W_{s}+\sum_{i} a_{i}\left(a_{i}+2\right) \leq 0 .
$$

Then if we show that $E_{s} \cdot W_{s}>0$, we must have a negative $a_{i}$, and then $x_{i} \in$ $\operatorname{Supp}\left(E_{s}\right)$. By construction $L=E_{s}+W_{s}, L \cdot E_{s}>0$ and

$$
L \cdot W_{s}=\left(L-2 E_{s}\right) \cdot L+L \cdot E_{s}=\left(\widetilde{L}-2 \widetilde{E}_{s}\right) \cdot \pi^{*} L+L \cdot E_{s}>0
$$

by (1). From the Hodge index theorem we get $E_{s} \cdot W_{s}>0$.

Now we need a result in [3], called the uniform multiplicity property. See also 6].

Lemma 3.1. Choosing $s$ and $E_{s}$ generally we can assume that the multiplicity of $E_{s}$ at every point of $Z$ is the same. 
Since for any $s$ there exists $x \in Z$ such that $x \in \operatorname{Supp}\left(E_{s}\right)$, by the uniform multiplicity property, we can choose $s$ and $E_{s}$ generally such that $Z \subset \operatorname{Supp}\left(E_{s}\right)$. For simplicity, we denote this divisor by $E$.

$Z \subset \operatorname{Supp}(E)$ implies that the multiplication by $E$ defines a map $\mathcal{O}_{X}(L-E) \rightarrow$ $\mathcal{O}_{X}(L) \otimes I_{Z}$. Since the cohomology group in Corollary 2.1 vanishes, this map lifts to an injective map $\mathcal{O}_{X}(L-E) \rightarrow V$. Thus, $\mathcal{O}_{X}(L-E)$ is a subsheaf of $V$.

It remains to prove that $V$ is unstable. This is equivalent to showing that

$$
(L-2 E)^{2}>0, \quad(L-2 E) \cdot L>0 .
$$

For the first inequality we consider the following exact sequence:

$$
0 \rightarrow \mathcal{O}_{X}(L-E) \rightarrow V \rightarrow \mathcal{O}_{X}(E) \otimes I_{Z^{\prime}} \rightarrow 0,
$$

for some zero dimensional scheme $Z^{\prime}$. Then $c_{1}(V)=L$ and $c_{2}(V)=(L-E) \cdot E+\left|Z^{\prime}\right|$, and by hypothesis we get

$$
(L-2 E)^{2}>4\left|Z^{\prime}\right|>0 .
$$

For the second one we note that

$$
\alpha=c_{1}^{2}(V)-c_{1}^{2}(V)+4 c_{2}(V)=4 c_{2}(V),
$$

and Proposition 2.3 gives the following:

$$
L \cdot E<2 c_{2}(V)
$$

Then

$$
L^{2}>4 c_{2}(V)>2 L \cdot E \text {. }
$$

\section{ACKNOWLEDGMENTS}

First the author would like to express his gratitude to Professor János Kollár for his constant support and many enlightening discussions. He would also like to thank Professor Robert Lazarsfeld, Luca Di Cerbo and the referee for constructive comments on the paper.

\section{REFERENCES}

[1] Bogomolov F., Holomorphic tensors and vector bundles on projective manifolds, Math. USSR Isvestija 13 (1979), 499-555. MR0522939 (80j:14014)

[2] Ein L., Lazarsfeld R., Global generation of pluricanonical and adjoint linear series on smooth projective threefolds, Journ. of Am. Math. Soc. 6 (1993), 875-903. MR.1207013 (94c:14016)

[3] Fernández del Busto G., Bogomolov instability and Kawamata-Viehweg vanishing, J. Alg. Geom. 4 (1995), 693-700. MR1339844 (96h:14062)

[4] Friedman R., Algebraic surfaces and holomorphic vector bundles, Universitext, Springer (1998). MR1600388 (99c:14056)

[5] Gieseker D., On a theorem of Bogomolov on Chern classes of stable bundles, Amer. J. Math. 101, no. 1 (1979), 77-85. MR527826 (80j:14015)

[6] Lazarsfeld R., Lectures on linear series, Complex Algebraic Geometry (Park City, UT, 1993), IAS/Park City Math. Series, vol. 3, Amer. Math. Soc., Providence, RI (1997), 161-219. MR:1442523 (98h:14008)

[7] Lazarsfeld R., Positivity in Algebraic Geometry II, Ergebnisse der Mathematik und ihrer Grenzgebiete (3), vol. 49, Berlin: Springer (2004). MR2095472 (2005k:14001b)

[8] Miyaoka Y., The Chern classes and Kodaira dimension of a minimal variety, Algebraic Geometry (Sendai 1985), Advanced Studies in Pure Mathematics, vol. 10, Kinokuniya, Tokyo; North Holland, Amsterdam (1987), 449-476. MR946247 (89k:14022) 
[9] Reid M., Bogomolov's theorem $c_{1}^{2} \leq 4 c_{2}$, Proc. Internat. Symposium on Alg. Geom., Kyoto (1977), Kinokuniya Book Store, Tokyo, 1978, 623-642. MR0578877 (82b:14014)

[10] Reider I., Vector bundles of rank 2 and linear systems on algebraic surfaces, Ann. Math. 127 (1988), 309-316. MR.932299 (89e:14038)

[11] Sakai F., Reider-Serrano's method on normal surfaces, Algebraic Geometry, Proceedings: L'Aquila 1988, Lect. Notes in Math., vol. 1417, Springer, 1990, 301-319. MR.1040564 (91d:14001)

[12] Serrano F., Extension of morphisms defined on a divisor, Math. Ann. 277 (1987), 395-413. MR891582(88f:14014)

Department of Mathematics, Princeton University, Princeton, New Jersey 085441000

E-mail address: gdi@math.princeton.edu 